\title{
Outdoor-to-indoor Propagation Characteristics of 1900 MHz Signals in Macro-cellular Environments for GSM and UMTS Systems
}

\author{
Hisham Elgannas ${ }^{*}$, Ivica Kostanic \\ Department of Electrical and Computer Engineering, Florida Institute of Technology, United States
}

Copyright (C) 2015 Horizon Research Publishing All rights reserved.

\begin{abstract}
Building penetration loss at $1900 \mathrm{MHz}$ bands in suburban environment is evaluated. The measurements are conducted in real Global System for Mobile Communications (GSM) and Universal Mobile Telecommunications System (UMTS) networks. Four buildings are studied aiming to provide first-order statistics of mobile signal coverage inside buildings. Results show that, on average, no significant signal band dependency has been confirmed. In general, UMTS-1900 MHz signals exhibit slight higher penetration loss values than GSM-1900 MHz signals. Analysis shows that the mean building penetration loss for all measured signals at the ground floor is about $16 \mathrm{~dB}$. The standard deviation of building penetration loss was about $4.5 \mathrm{~dB}$ for wideband signals and $2.5 \mathrm{~dB}$ for narrowband signals. Results show also that, the average rate of change in building penetration loss with height is $0.95 \mathrm{~dB}$ per meter for wideband signals versus $0.65 \mathrm{~dB}$ per meter for narrowband signals.
\end{abstract}

Keywords GSM-1900MHz, UMTS-1900MHz, Outdoor-to-indoor Propagation, Building Penetration Loss, Floor Height Gain

\section{Introduction}

In the most recent surveys, it has been reported that a high proportion of cellular traffic originates from inside buildings [1]. Therefore, more significance is given to indoor environment to meet the increasing demand for high speed data services and capacity. Current wireless networks such as LTE and LTE Advanced offer flat rate for smart devices in a form of phones, laptops, or multimedia devices. There is also requirement of other services like position information of the mobile user inside large buildings, malls, hospitals, air ports, public places, factories and emergency services. However, provisioning of reliable radio coverage for mobiles inside buildings is a challenging task. For example, to have satisfactory operation of a mobile system in indoor environment, the outdoor base stations should be installed so that maximum capacity and minimum co-channel interference are achievable.

In outdoor-to-indoor scenarios, providing high speed data services to users inside building is directly proportional to the available Signal to Interference and Noise Ratio (SINR) throughout the building. Like outdoor and indoor channels, the parameters of outdoor-to-indoor channel are statistical in nature. Therefore, an extensive measurements campaign and detailed analysis is of a fundamental importance for a reliable characterization of the main aspects of this channel [2].

Several studies regarding measurements-based outdoor-to-indoor building penetration loss characterization and modeling have been published [2-13]. The buildings examined in these publications range from small residential buildings to large office buildings where penetration loss at frequencies up to $8 \mathrm{GHz}$ has been studied. However, none of the above mentioned publications has characterized the outdoor-to-indoor propagation of GSM signals versus UMTS signals that operate at same frequency. In the previous work [3], the frequency dependency of the building penetration loss for GSM-850 MHz and GSM-1900 MHz signals is investigated. In [3], a maximum difference of $2.8 \mathrm{~dB}$ in building penetration loss is observed when signal frequency band increases from $850 \mathrm{MHz}$ to $1900 \mathrm{MHz}$.

In like manner, the main focus of the study presented in this paper is of the building penetration loss as a function of the signal bandwidth. GSM- $1900 \mathrm{MHz}$ as a narrowband signal, and UMTS-1900 MHz as a wideband signal, are considered in this investigation. Extensive measurements were conducted in real GSM and UMTS networks in order to provide fair comparison of the building penetration loss characteristics of GSM and UMTS signals. A detailed analysis is performed to present results with statistical significance.

The building penetration loss data are presented graphically and coupled with site-specific information.

The paper is organized as follows. Section 2 describes the 
experimental hardware used, the environment where the buildings are located, the data collection and processing. The most important aspects of the building penetration loss and summary of most interesting results are presented in Section 3. Finally, section 4 provides conclusion of this study.

\section{Measurement Setup}

\subsection{Description of Measurement Site}

The four buildings measured are located on the campus area of Florida Institute of Technology, and represent typical university buildings. They are multi-story buildings made of hard materials with office spaces and classrooms inside.

The measurements are designed to test the outdoor to indoor building penetration loss for narrowband and wideband signals at GSM-1900 MHz and UMTS-1900 $\mathrm{MHz}$ frequency bands. The measurements are performed on real commercial networks. Macro-cell base stations serving the area have antennas located well above the average buildings heights. Buildings are typically detached office and educational halls with two to seven floors. To avoid any dependency on the particular configuration of BSs around buildings, six BSs were examined. Locations of buildings where measurements were conducted are shown in Figure 1. The figure also shows the relative position of the examined buildings with regard to the located GSM-1900MHz and UMTS-1900MHz base stations.

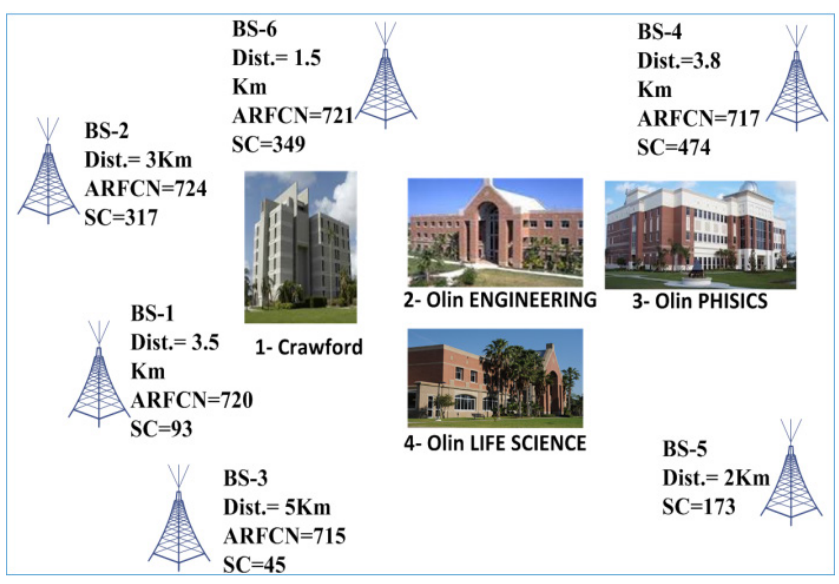

Figure 1. Locations of examined buildings and base stations

\subsection{Building Description}

The examined buildings are Crawford tower (CRAW), Olin Engineering complex (O.ENG), Olin Life Sciences (O.LS), and Olin Physical Science Center (O.PSC). These buildings include specialized research and teaching laboratories, classrooms, and conference rooms. Offices on all floors of all buildings were crowded with typical office furniture. The teaching or research laboratories contained experimental equipment according to the specialized demand of each area of study. The floor height in all buildings is about $4 \mathrm{~m}$. A brief description of the measured buildings is provided in Table 1 .

Table 1. Brief description of 4 measured buildings

\begin{tabular}{|c|c|c|c|c|c|}
\hline buld. & $\begin{array}{c}\text { Floor } \\
\text { area }\end{array}$ & $\begin{array}{c}\text { External } \\
\text { wall }\end{array}$ & $\begin{array}{c}\text { Windows } \\
\text { percentage }\end{array}$ & $\begin{array}{c}\text { Const. } \\
\text { date }\end{array}$ & $\begin{array}{c}\text { No. } \\
\text { of } \\
\text { floors }\end{array}$ \\
\hline CRAW & $360 \mathrm{~m}^{2}$ & $\begin{array}{c}\text { concrete } \\
\text { masonry }\end{array}$ & $\begin{array}{c}10 \% \text { of } \\
\text { building } \\
\text { sides. }\end{array}$ & 1961 & 7 \\
\hline O.PSC & $\begin{array}{c}2300 \\
\mathrm{~m}^{2}\end{array}$ & $\begin{array}{c}\text { brick and } \\
\text { concrete } \\
\text { masonry }\end{array}$ & $\begin{array}{c}16 \% \text { of } \\
\text { building } \\
\text { sides. }\end{array}$ & 2005 & 4 \\
\hline O.ENG & $\begin{array}{c}2100 \\
\mathrm{~m}^{2}\end{array}$ & $\begin{array}{c}\text { brick and } \\
\text { concrete } \\
\text { masonry }\end{array}$ & $\begin{array}{c}28 \% \text { of } \\
\text { building } \\
\text { sides. }\end{array}$ & 1999 & 3 \\
\hline $\begin{array}{c}2000 \\
\mathrm{~m}^{2}\end{array}$ & $\begin{array}{c}\text { brick and } \\
\text { concrete } \\
\text { masonry }\end{array}$ & $\begin{array}{c}22 \% \text { of } \\
\text { building } \\
\text { sides. }\end{array}$ & 1999 & 2 \\
\hline
\end{tabular}

\subsection{Measurement Procedure Description}

The penetration loss in the study is defined as the difference in mean received signal level between the measurement obtained inside and a reference signal level measured outside near the unobstructed walls of the building [3]. In order to provide reliable references for the respective buildings, over two hundred data points at different locations around each building have been considered. For indoor measurements, the floor area has been divided into small squares and the area of each is $2 \mathrm{~m}^{2}$. With this setup, more than 8 thousand in-building locations using Agilent Digital Receivers have been measured. The Broadcast Control Channels (BCCH) of 6 surrounding GSM-1900MHz base stations and the scrambling codes (SC) of 6 UMTS-1900MHz signals transmitting from same base stations were measured. The receivers perform measurement of received signal levels for all frequency channels simultaneously. The analyzer displays the amplitude of the Absolute Radio Frequency Channel Number (ARFCNs) and the scrambling codes (SC) for all measurements with the corresponding power levels in $\mathrm{dBm}$ and the associated decoded Base Station Identity Codes (BSIC).

The data collection system setup (for both indoor and outdoor locations) is also shown in Figure 2. The receiving antennas are mounted on the top of a wooden mast at height of $1.5 \mathrm{~m}$ above the examined floor. To avoid undesirable deviations of the received signal level due to body losses and antenna orientation in some static positions, the receiving antenna was slowly tilted and moved within the measured location to average out all these effects.

All locations of the measurement runs are marked on the respective floor plan. In each location, the measurement course lasted 4 minutes which is enough time to get sufficient number of samples for each measured channel. With this setup, the collected data per specific point for 
each base station allow for determination of the statistical properties of the received signal level at each data point. Kolmogrov-Smirnov test shows that the measurements are distributed log-normally.
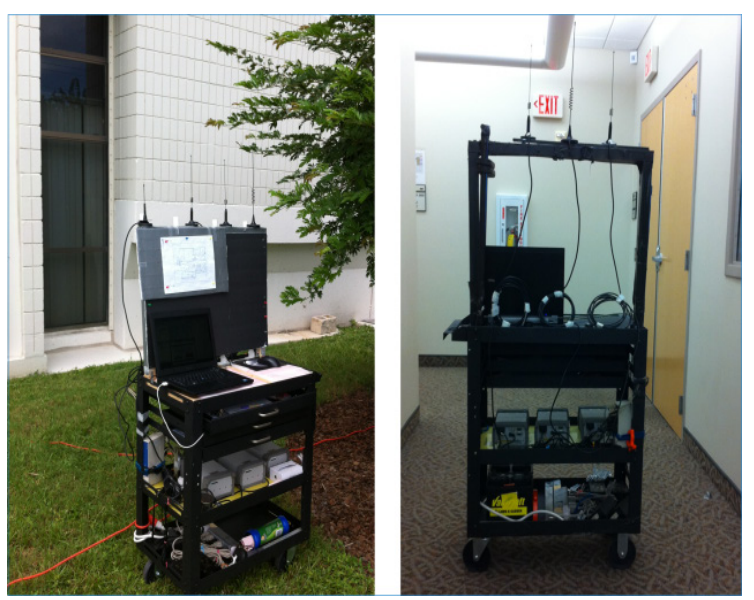

Figure 2. The equipment used and the data collecting system in two different locations. Outdoor data point (left), indoor data point (right).

In order to provide site-specific information about the radio coverage inside buildings, color coded plots were used. Figure 4 shows a sample scatter plot of received power level measurements on ground floor of Crawford building (left). Dots symbolize location of successive measurement points and the color of each dot indicates basic received signal level in $\mathrm{dBm}$. An interpolated view of the same measurements is presented on the right side of Figure 4. The interpolated view in Figure 4 was created by using spatial interpolation. Inverse Distance Weighting (IDW) interpolation method was used to predict the expected average signal level in places where measurements were not possible.
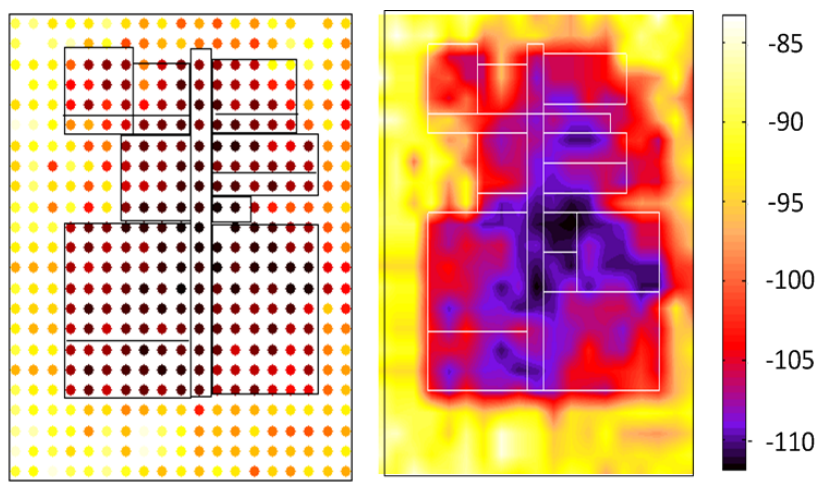

Figure 4. Scatter plot and its interpolated view of a radio coverage inside building

\section{Measurement Results and Data Analysis}

One of the most important parameters in characterizing the outdoor-to-indoor propagation is the building penetration loss. This loss can be simply defined as the additional loss that radio signal transmitted from outdoor base station undergoes as it propagates into buildings. There are several factors that have shown to have significant effect on the building penetration loss values [14]. In the next subsections, the effects of the main factors influenced the measured building loss are discussed in more detail. These factors include signal bandwidth, presence of line-of-sight conditions, and floor height gain.

\subsection{Average Building Penetration Loss}

Different ways of calculating building penetration loss are found in the literature [9] and [15]. In the previous study [3], the building loss was calculated as the difference between the average received power at a reference point outside building (measured at base station side of the building) and the individual average of the received power at all specified data points inside building. This approach was adopted in this study as well. The approach is practical and readily applied to outdoor-to-indoor propagation models that are used to predict the in-building signal power level. Typically, those models predict the propagation loss at the street levels and add the building penetration loss as additional loss.

Table 2 summarizes the average building loss and the standard deviation of the four measured buildings. It is obvious from Table 2 that both GSM and UMTS channels operating at $1900 \mathrm{MHz}$ encounter nearly the same mean building penetration loss value.

Table 2. Average and standard deviation values of building penetration losses for GSM-1900 MHz and UMTS-1900MHz channels at the ground floor of the four measured buildings

\begin{tabular}{|c|c|c|c|c|c|}
\hline$\#$ & Building & \multicolumn{2}{|c|}{ GSM-1900 MHz } & \multicolumn{2}{c|}{ UMTS-1900 MHz } \\
\hline & & Mean & STD & Mean & STD \\
\hline 1 & CRAW & 14.1 & 3.0 & 13.7 & 4.5 \\
\hline 2 & O.ENG & 18.1 & 2.47 & 18.2 & 4.8 \\
\hline 3 & O. PSC & 22.15 & 1.5 & 19 & 4.2 \\
\hline 4 & O. LS & 12.8 & 2.3 & 12.4 & 4 \\
\hline
\end{tabular}

\subsection{The Impact of Channel Bandwidth}

Figure 5 shows a comparison plot of the mean and the standard deviation of the penetration loss obtained in four measured buildings. Eight signals transmitted from four different base stations with GSM-1900MHz and UMTS-1900MHz are considered.

It is clear from Figure 5 that the UMTS signals experience nearly the same mean and standard deviation of loss within each individual building. This consistency is due to the fact that all measured UMTS signals operate at the same carrier frequency but they are separated by different scrambling codes. On contrary, GSM signals transmitted from different base stations encounter different mean and standard deviation values of building penetration loss in each individual building. 
It is noticeable also in Figure 5 that the building penetration loss of all measured buildings is slightly higher for wideband signals than narrowband signals for three different base stations. However, three buildings (CRAW, O.PSC, and O.LS) exhibit higher penetration loss for narrowband signals that are transmitted from BS1 than the wideband signals received from the same base station.
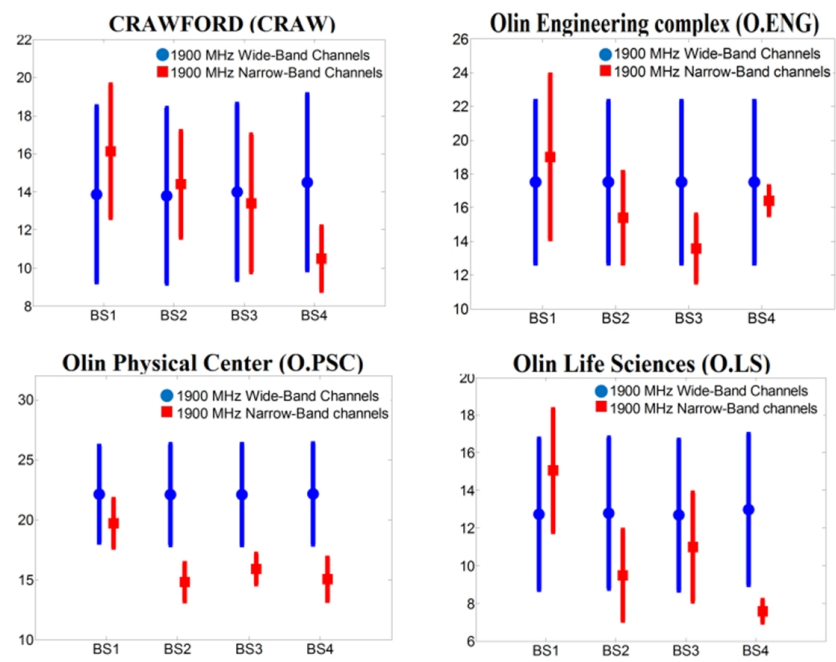

Figure 5. Computed building penetration mean and standard deviation for UMTS-1900 MHz versus GSM-1900 MHz channels in the four measured buildings.

Figure 6 shows a sample plot of the difference in building penetration loss between narrowband signals (on left) and wideband signals (on right) in two different building (O.LS building on top and O.PSC on bottom). This plot represents an example of building loss distribution in O.ENG, O.LS, and O.PSC buildings.
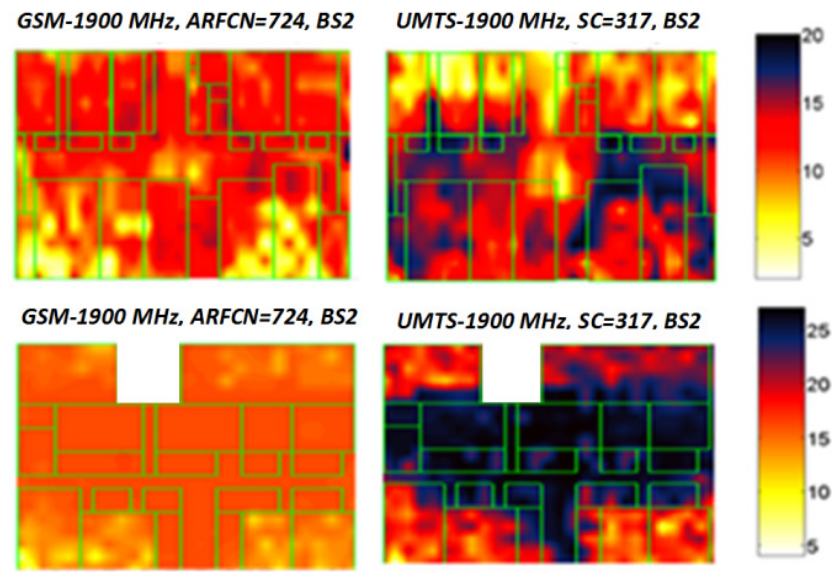

Figure 6. Measured penetration loss in O.LS building (top) and O.PHY building (bottom) of GSM-1900MHz channels (left) versus UMTS-1900 $\mathrm{MHz}$ channels (right)transmitting from same base station (BS2).

However, results also showed that both wideband and narrowband signals that are transmitting from same base station may encounter nearly the same average building penetration loss. In this case, where no significant signal band dependency rises, it is useful to compare the building penetration loss distribution of both narrowband and wideband signals. For example, the received signals at ground floor of Crawford building from BS2 and BS3 undergo a building loss of about $14 \mathrm{~dB}$ (see Figure 5). Figure 7 shows a comparison plot of the building penetration loss of the four different signals (narrowband and wideband) that are received from BS2 and BS3.
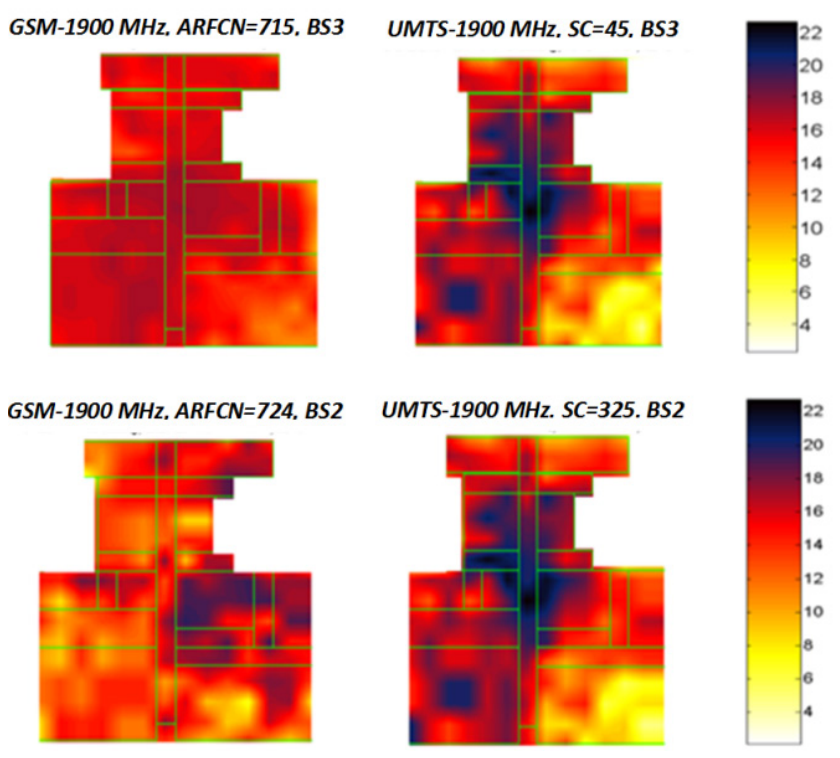

Figure 7. Measured penetration loss of four channels transmitting from two different base stations. Two channel are in GSM-1900MHz band (left) and two in UMTS-1900MHz band (bottom)

On the top of Figure 7, the building loss map of GSM signal $\mathrm{ARFCN}=715$ is compared with UMTS signal $\mathrm{SC}=45$. Both signals are transmitted by BS3 which has relative position with the measured building as shown in Figure 1. A similar comparison is made on the bottom of Figure 7 between GSM-transmitter signal $\mathrm{ARFCN}=724$ and UMTS-transmitter signal $\mathrm{SC}=93$. Figure 7 emphasizes the importance of reporting the standard deviation values of building penetration losses of the different signals. The relative high standard deviation values of the building loss may be attributed to the presence of different propagation mechanisms that influence together the overall field. For example, layout of measured building, surrounding building, and trees may create diffracted, reflected, and/or scattered different transmission paths. These additional paths may contribute in increase the received signal power in some locations and/or decrease it in other locations. For indoor measurements, the type of furniture, the type of equipment used in labs, type of internal partitions, and body loss of people moving around may also influence the mean and the standard deviation of the building loss values, especially at the center region of each building.

\subsection{The Impact of the Presence of line-of-sight}

In outdoor-to-indoor propagation, the LOS condition is not related to presence or absence of direct path between 
transmit antenna and receive antenna [2]. Instead, the LOS path is the direct path between the outdoor antenna and the immediate exterior environment to the building.

In the presence study, the relative position of each transmitter (base station) and the associated channel of each base station are well known. However, determination of accurate transmission condition for each floor in the measured buildings with respect to specific transmitter, especially in Macro-cellular environment, is not always possible. One way to investigate the transmission condition is to plot and analyze the radio coverage of each measured signal in the studied buildings and relate results to the relative position of each base station to those buildings (shown in Figure 1).

Results show that the LOS condition has significant effects on the standard deviations of both indoor and outdoor measurements. It has also obvious influence on penetration distance of measured signals (penetration depth). These results confirm obtained observations reported in previous work for GSM signals [2], [3], and [8].

Outdoor measurements in locations placed in both the illuminated and non-illuminated facades of the four measured buildings show relatively consistent higher values of standard deviations for UMTS signals. For GSM $1900 \mathrm{MHz}$ signals, the standard deviation of outdoor measurements takes values between $0.6 \mathrm{~dB}$ and $4.9 \mathrm{~dB}$, while, it ranges between $4 \mathrm{~dB}$ and $4.8 \mathrm{~dB}$ for UMTS 1900 MHz signals.

On the other hand, indoor measurements confirmed an expected decrease of loss within LOS-areas inside building when compared to NLOS-areas. At same antenna height and with presence of windows and sufficient clearance to the operating base station, an average difference of $7 \mathrm{~dB}$ in building loss between LOS-areas and NLOS-areas inside buildings has been observed.

Figure 8 shows a comparison plot of building penetration loss of the ground floor of O.PSC building. Four GSM 1900 $\mathrm{MHz}$ channels that are transmitting from different base stations are considered in the plot. It is assumed here that the ground floor of O.PSC building has four different transmission conditions with the measured GSM signals.

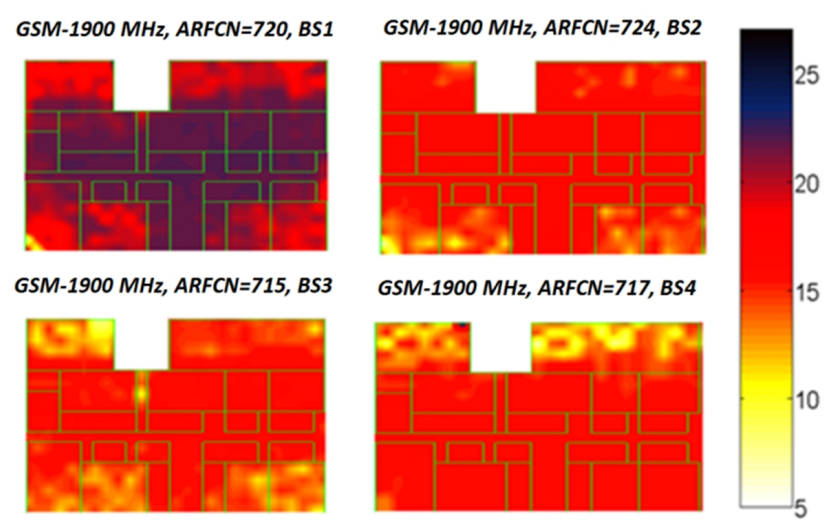

Figure 8. Building penetration loss plots of four different GSM 1900 $\mathrm{MHz}$ transmitters in different transmission conditions for the ground floor of O.PHY building .
It is clear from Figure 8 that the transmission condition has noticeable influence on the mean and standard deviation of building penetration loss. On the top left corner of Figure 8 , the transmission path between $\mathrm{BS} 1$ and the respective ground floor is partially blocked by O.ENG building. As a result of the NLOS condition, the building penetration losses take higher values in comparison with the other transmission conditions. In the other plots of Figure 8, the effect of the direct transmission path (LOS) is obvious on in-building areas, especially at the base station side of the respective building. In such cases, direct transmission paths encounter higher diffraction than other paths which in turn causes significant fluctuations in the received signal power. This explains the relatively higher standard deviation values observed at LOS-areas inside buildings.

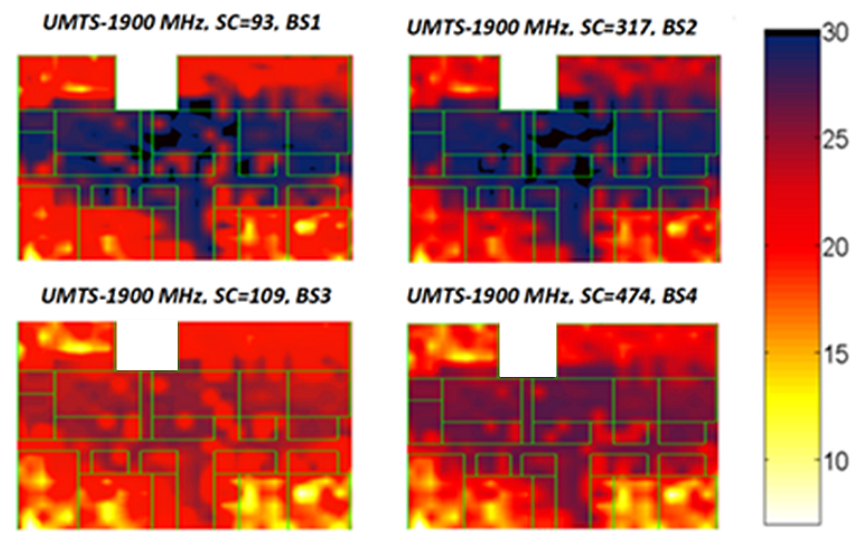

Figure 9. Building penetration loss plots of four different UMTS 1900 $\mathrm{MHz}$ transmitters in different transmission conditions for the ground floor of O.PHY building .

Similar comparison plot for the ground floor of O.PSC building that are subjected to different transmission conditions of UMTS 1900 signals is shown in Figure 9.It is noticeable from Figure 9 that the distribution of building penetration loss of the different UMTS signals is nearly same for all signals. It is clear too that BS3 and BS4 provide better radio coverage than $\mathrm{BS} 1$ and $\mathrm{BS} 2$, due to more favourable propagation conditions that BS3 and BS4 have with the respective building. As mentioned, the transmission paths between BS1 and BS2 and the respective floor are partially blocked by O.ENG building.

\subsection{The Impact of Floor Height Gain and Penetration Depth}

In order to calculate the building penetration loss at high receive-antenna heights, one needs to relate indoor measurements to outdoor reference measurements. However, conducting outdoor measurements at high building floors is not always possible. Therefore, the floor height gain model used to define the decrease in building penetration loss values at higher building levels with respect to outdoor reference measurements taken at ground floor.

Regression analysis is used to evaluate the floor height 
gain effect. Figure 10 shows a plot of a line that best fits the indoor measurements for UMTS signals in Crawford building. To eliminate other factors that may affect the received power levels of different signals, only in-building locations at the base station side are considered.

The $\mathrm{x}$ axis of this plot represents the absolute heights of the receive antenna in meters while the power levels are represented in $\mathrm{dBm}$ on the $\mathrm{y}$ axis. It may be seen clearly that as the receive antenna height increases, a steadily increase in the received power levels becomes higher as well. Therefore, the building penetration loss values decreases with an increase of the received antenna's height.

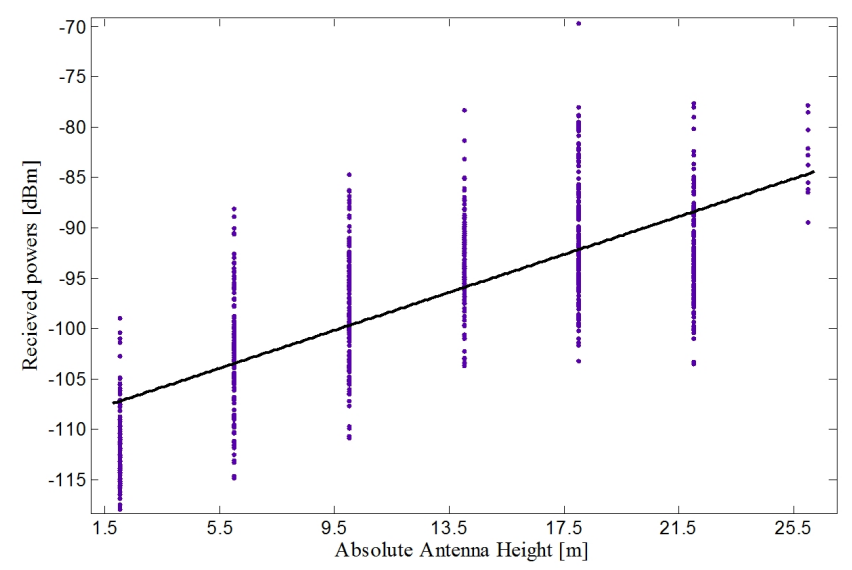

Figure 10. Average received power levels of UMTS channels versus receiver heights in high-rise building together with best fit line.

For all measurements in Crawford building, the floor height gain ranges between $0.49 \mathrm{~dB} / \mathrm{m}$ and $0.60 \mathrm{~dB} / \mathrm{m}$ for GSM-1900 MHz signals, while, this gain has consistent value of $0.95 \mathrm{~dB} / \mathrm{m}$ for UMTS- $1900 \mathrm{MHz}$ signals. These results are in good agreement with the findings reported in [2-7]. However, the effect of floor height was not reliable as pronounced in the other measured buildings (O.ENG, O.LS, and O.PSC). In these buildings, the difference in received signal power levels between ground floor and the highest floor was clear but not consistent for GSM channels. In contrast, the floor height gain for UMTS signals has 0.8 $\mathrm{dB} / \mathrm{m}$ rate of change in those three buildings.

This inconsistent effect of the floor height on the penetration loss in those buildings can be attributed to different factors. Examples of such factors are the transmission condition, the relatively low heights of these buildings with respect to the heights of macrocellular base stations, and the relatively large floor areas of these buildings in comparison with Crawford building. Therefore, the floor height gain may not be observable in all propagation scenarios.

The other observed influence of the floor height is the increase in penetration depth of radio signal as it propagates through building. When mobile station moves to higher floors, the outdoor-to-indoor transmission condition may also change. Then, the chance to receive direct transmission paths (LOS path) from different transmitters increases.

The impact of floor height on the received power level, and therefore on the building penetration depth, is depicted in Figure 11. Three plots represent the radio coverage map of UMTS signal in three different floor heights of Crawford building are considered.
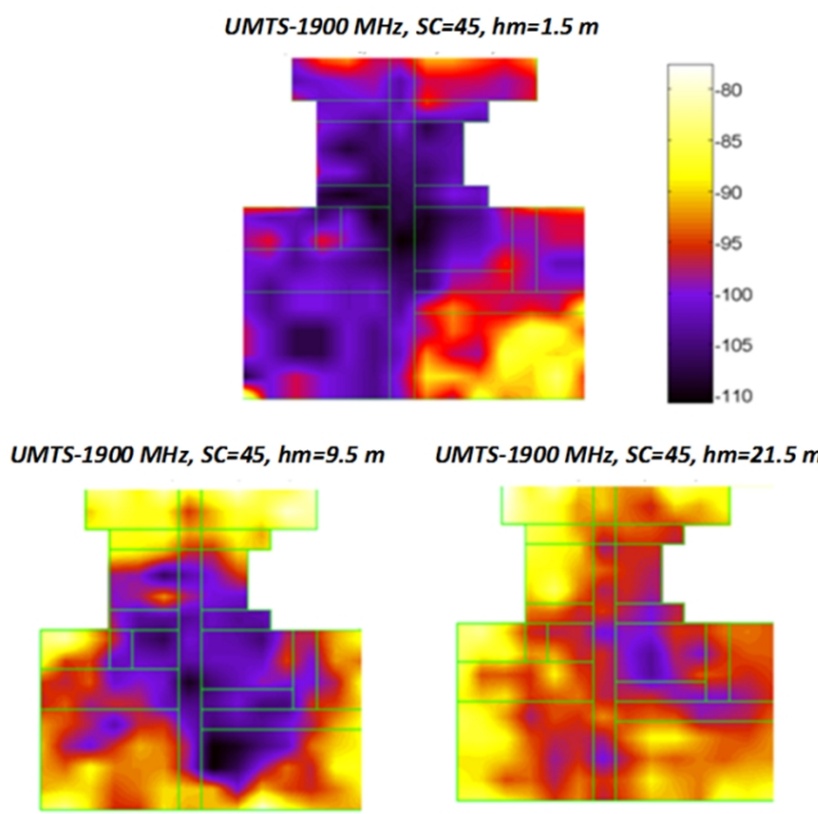

Figure 11. Increasing in the penetration depth of the signal propagation for one base station (BS3) within three different floors inside Crawford building

The measurements of the received power levels of UMTS transmitter (BS3) in $1^{\text {st }}$ floor is plotted on the top of Figure 11. The radio coverage map of the $3^{\text {rd }}$ floor is shown in the lower left whereas the coverage map of the $6^{\text {th }}$ floor is shown in the lower right.

The effect of floor height, and therefore the LOS condition on the building penetration depth is obvious in Figure 11. The penetration depth increases in areas that are subjected to a clear line of sight with the operating base station. In such areas, building penetration loss becomes more linear which in turn makes the process of modeling this loss more practical. A possible explanation for this observation is that at higher floors, the effect of other attenuation factors is limited to the minimum with the presence of LOS transmission condition.

\section{Summary and Conclusions}

In this paper, the building penetration loss in macro-cellular environment is investigated for mobile signals of PCS $1900 \mathrm{MHz}$ and UMTS $1900 \mathrm{MHz}$ communication systems. The building loss was evaluated for 4 office buildings located in suburban area. The measurement data were statistically analysed, and the main characteristics for building penetration loss were shown. On average, the building loss was $16 \mathrm{~dB}$ for both narrowband and wideband signals with higher standard deviation of 4.5 $\mathrm{dB}$ for wideband signals. For all measurements, signal band 
dependency was noticeable for a number of base stations.

The received UMTS signals from those base stations penetrates walls less easily, while the penetration loss of their GSM signals is less sever. Additionally, UMTS signals encounter consistent building losses in each measured building with standard deviation of $4.5 \mathrm{~dB}$. Results show also that UMTS signals have consistent $0.95 \mathrm{~dB} / \mathrm{m}$ rate of change in building penetration loss as receivers move upwards to higher floors. This floor height gain has an average value of $0.56 \mathrm{~dB}$ for GSM $1900 \mathrm{MHz}$ signals. Moreover, plots of the radio coverage of different signals confirmed significant influence of the line-of-sight presence on both the building loss values and the signal penetration depth.

Finally, the results obtained in this study are in good agreement with the results from similar studies. This research will serve as a base for future studies concerned with modeling of narrowband and wideband outdoor-to-indoor channels at $1900 \mathrm{MHz}$ band.

\section{REFERENCES}

[1] De, la R. G, Andres A Glazunov, Ben Allen, LTE-Advanced and Next Generation Wireless Networks: Channel Modelling and Propagation. Chicago: Wiley, 2013.

[2] J. Berg, 4.6 building penetration: Digital mobile radio toward future generation systems. Brussels, Belgium, 1999.

[3] H. Elgannas, I. Kostanic, Outdoor-to-Indoor Propagation Characteristics of $850 \mathrm{MHz}$ and $1900 \mathrm{MHz}$ Bands in Macro -Cellular Environments, accepted for publication in World Congress on Engineering and Computer Science 2014 (WCECS 2014), San Francisco, USA, 23-25 October, 2014.

[4] E. H. Walker, penetration of radio signals into buildings in the cellular radio environment, The bell systems Technical Journal, vol. 62, No.9, November 1983.

[5] W. J. Tanis, II and G. J. Pilato, Building penetration characteristics of $880 \mathrm{MHz}$ and $1922 \mathrm{MHz}$ radio waves, in Vehicular Technology Conference, 1993., 43rd IEEE, 1993, pp. 206-209.

[6] S. Aguirre, L. H. Loew, and L. Yeh, Radio propagation into buildings at 912,1920, and $5990 \mathrm{MHz}$ using microcells, in Universal Personal Communications, 1994. Record., 1994
Third Annual International Conference on, 1994, pp. 129-134.

[7] A. M. d. Turkmani, J.D. Parsons, Measurement of building penetration loss on radio signals at 441,900 and $1400 \mathrm{MHz}$, Electronic and Radio Engineers, Journal of the Institution of, vol. 58, pp. S169-S174, 1988.

[8] D. M. Rose and T. Kurner, Outdoor-to-indoor propagation ; Accurate measuring and modelling of indoor environments at 900 and $1800 \mathrm{MHz}$, in Antennas and Propagation (EUCAP), 2012 6th European Conference on, 2012, pp. $1440-1444$.

[9] A.F. De Toledo, A. Turkmani, D. Parsons, Estimating coverage of radio transmission into and within buildings at 900, 1800, and $2300 \mathrm{MHz}$, Personal Communications, IEEE, vol. 5, pp. 40-47, 1998.

[10] E. F. T. Martijn and M. H. A. J. Herben, Characterization of radio wave propagation into buildings at $1800 \mathrm{MHz}$, Antennas and Wireless Propagation Letters, IEEE, vol. 2, pp. 122-125, 2003.

[11] J. Medbo, J. Furuskog, M. Riback, J. E. Berg, Multi-frequency path loss in an outdoor to indoor macrocellular scenario, in Antennas and Propagation, 2009. EuCAP 2009. 3rd European Conference on, 2009, pp. 3601-3605.

[12] H. Okamoto, K. Kitao, and S. Ichitsubo, Outdoor-to-Indoor Propagation Loss Prediction in 800-MHz to 8-GHz Band for an Urban Area, Vehicular Technology, IEEE Transactions on, vol. 58, pp. 1059-1067, 2009.

[13] C. Kin Lien, A. Anggraini, T. Kaiser, T. Kurner, Outdoor-to-indoor propagation loss measurements for broadband wireless access in rural areas, in Antennas and Propagation (EUCAP), Proceedings of the 5th European Conference on, 2011, pp. 1376-1380.

[14] S. Stavrou and S. R. Saunders, Factors influencing outdoor to indoor radio wave propagati on, in Proc. 12th Int. Conf. Antennas Propag. (ICAP 2003), Mar. 31-Apr. 3 2003, pp. 581-585.

[15] D. C. Cox, R. R. Murray, and A. W. Norris, Measurements of $800 \mathrm{MHz}$ radio transmission into buildings with metallic walls, Bell Syst. Tech. J., vol. 62, no. 9, pp. 2695-2717, Nov. 1983.

[16] L Ferreira, M Kuipers, C Rodrigues, Characterization of Signal Penetration into Buildings for GSM and UMTS, in Proc. 3rd Int. Symp. on Wireless Communication Systems, Valencia, Spain, Sep. 2006, pp. 63-67. 